Pacific Journal of Mathematics

PEIRCE DECOMPOSITION IN SIMPLE LIE-ADMISSIBLI 


\title{
PEIRCE DECOMPOSITION IN SIMPLE LIE-ADMISSIBLE POWER-ASSOCIATIVE RINGS
}

\author{
William E. Coppage
}

\section{The main result is}

Theorem. If $A$ is a simple Lie-admissible power-associative ring with characteristic prime to six, and if $A$ has an idempotent $e$ relative to which $A$ has a Peirce decomposition such that $A_{00}=0$, then either $e$ is a unity element of $A$ or $A \cong B$, where $B$ is a three-dimensional algebra having a basis $\{e, x, y\}$ such that $e^{2}=e, e x=x, y e=y, x y=-y x=e$ and $x e=e y=x^{2}=y^{2}=0$.

If $A$ is a simple Lie-admissible power-associative ring then $A$ belongs to a class of rings which includes associative rings, Lie rings, commutative power-associative rings, Jordan rings, anti-flexible rings, rings of type $(\gamma, \delta)$ and others. Lie rings do not have idempotent elements, and simple $(\gamma, \delta)$ rings with an idempotent $e \neq 1$ have been shown $[2,3,4,5,6,8]$ to be associative. Thus if $A$ has an idempotent element $e \neq 1$ then $A$ belongs to a class which includes rings of the associative, commutative power-associative, and antiflexible types. Assuming that $A$ has an idempotent $e$ satisfying,

$$
(e, e, x)=(e, x, e)=(x, e, e)=0,
$$

suffices to establish a Peirce decomposition,

$$
A=A_{11}+A_{10}+A_{01}+A_{00},
$$

where $A_{i j}=\{x \in A \mid e x=i x, x e=j x\}$ for $i, j=0,1$. This assumption eliminates the possibility that $A$ is commutative, for then $A_{10}=A_{01}=0$, so [2] $A=A_{11} \oplus A_{00}$ and simplicity implies that $A=A_{11}$, hence $e$ is a unity element for $A$.

The class of rings under consideration does contain members which are not associative. Kosier [7] has given examples of simple Lieadmissible power-associative finite-dimensional algebras, the so-called anti-flexible algebras. These have the property that $A=A_{11}+A_{00}$ in every Peirce decomposition.

There are no rings with unity element, 1 , which possess a Peirce decomposition with respect to an idempotent $e \neq 1$ in which $A_{00}=0$. This is because $1-e \in A_{00}$.

The algebra $B$ of our theorem was introduced in [9]. It has the property that $B^{(-)}$is a simple Lie algebra.

The associator, $(x, y, z)=(x y) z-x(y z)$, and the commutator, $[x, y]=x y-y x$, are functions which, defined on any ring, are linear 
in each variable and related by the identity,

$$
[x y, z]+[y z, x]+[z x, y]=(x, y, z)+(y, z, x)+(z, x, y) .
$$

A Lie-admissible ring satisfies,

$$
[x y-y x, z]+[y z-z y, x]+[z x-x z, y]=0,
$$

and a power-associative ring whose characteristic is prime to two satisfies

$$
[x y+y x, z]+[y z+z y, x]+[z x+x z, y]=0,
$$

hence in the ring $A$ the function

$$
\begin{aligned}
H(x, y, z) & =(x, y, z)+(y, z, x)+(z, x, y) \\
& =[x y, z]+[y z, x]+[z x, y]
\end{aligned}
$$

is identically zero. Also, the fourth-power-associativity identities $\left(x^{2}, x, x\right)=0$ and $\left(x, x, x^{2}\right)=0$ may be linearized to yield functions $P(a, b, x, y)=\sum(a b, x, y)$ and $Q(a, b, x, y)=\sum(a, b, x y)$ which are identically zero. The $\sum$ here in both cases indicates a sum to be taken over the twenty-four permutations of $a, b, x$ and $y$.

We will use - as well as juxtaposition in denoting products, with juxtaposition taking precedence. Thus $a \cdot b c=a(b c)$.

Lemma. Let $A$ be a ring whose characteristic is prime to six and in which the functions $H, P$ and $Q$ vanish identically. Suppose $A$ contains an idempotent $e$ relative to which $A$ has a Peirce decomposition. If $a_{m n}$ denotes the component of an element $a$ in the module $A_{m n}$ then

$$
\begin{gathered}
x_{i i} y_{j j}=0, \\
x_{i i} y_{i i}+y_{i i} x_{i i} \in A_{i i}, \\
x_{i i}^{2} \in A_{i i}, \\
x_{i i} y_{i j}=\left(x_{i i} y_{i j}\right)_{i j}+\left(x_{i i} y_{i j}\right)_{j j} \in A_{i j}+A_{j j}, \\
y_{i j} x_{i i}=\left(x_{i i} y_{i j}\right)_{j j} \in A_{j j}, \\
y_{j i} x_{i i}=\left(y_{j i} x_{i i}\right)_{j i}+\left(y_{j i} x_{i i}\right)_{j j} \in A_{j i}+A_{j j}, \\
x_{i i} y_{j i}=\left(y_{j i} x_{i i}\right)_{j j} \in A_{j j}, \\
x_{i j} y_{i j}=y_{i j} x_{i j} \in A_{i i}+A_{j j}, \\
x_{i j} y_{j i} \in A_{i i}+A_{j j}, \\
x_{i i} y_{i i} \in A_{i i}+A_{j j},
\end{gathered}
$$




$$
\begin{gathered}
{\left[A_{i j}^{2}, A_{i i}+A_{j i}+A_{j j}\right]=0,} \\
{\left[A_{i j} A_{j i}, A_{j j}\right]=0,} \\
{\left[A_{j i} A_{j j}, A_{i j}\right]=0,} \\
{\left[A_{j j} A_{i j}, A_{j i}\right]=0,} \\
a_{i i} \cdot x_{i j} y_{i j}=x_{i j} y_{i j} \cdot a_{i i}=(1 / 2)\left(\left(a_{i i} x_{i j} \cdot y_{i j}\right)_{i i}+\left(a_{i i} y_{i j} \cdot x_{i j}\right)_{i i}\right), \\
a_{i i} \cdot x_{j i} y_{j i}=x_{i j} y_{i j} \cdot a_{i i}=(1 / 2)\left(\left(x_{j i} a_{i i} \cdot y_{j i}\right)_{i i}+\left(y_{j i} a_{i i} \cdot x_{j i}\right)_{i i}\right), \\
\left(a_{i i}\left(x_{i j} y_{j i}+y_{j i} x_{i j}\right)\right)_{i i}=\left(a_{i i} x_{i j} \cdot y_{j i}\right)_{i i}+\left(y_{j i} a_{i i} \cdot x_{i j}\right)_{i i},
\end{gathered}
$$

and

$$
\left(\left(x_{i j} y_{j i}+y_{j i} x_{i j}\right) a_{i i}\right)_{i i}=\left(y_{j i} \cdot a_{i i} x_{i j}\right)_{i i}+\left(x_{i j} \cdot y_{j i} a_{i i}\right)_{i i} \text {. }
$$

Proof of the lemma. Identities (5), (6) and (7) are derived in [1] using only the fact that the functions $H, P$ and $Q$ vanish identically. All of the identities are obtained by relatively straightforward substitution of elements into $H, P$ and $Q$. Due to the excessive length of many of the computations involved we leave the proofs to the reader.

Since our theorem hypothesizes that $A_{00}=0$ the multiplicative properties stated in (8) through (14) of the preceding lemma can be more compactly exhibited in our case by the module multiplication table:

\begin{tabular}{c|c|c|c} 
& $A_{11}$ & $A_{10}$ & $A_{01}$ \\
\hline$A_{11}$ & $A_{11}$ & $A_{10}$ & 0 \\
\hline$A_{10}$ & 0 & $A_{11}$ & $A_{11}$ \\
\hline$A_{01}$ & $A_{01}$ & $A_{11}$ & $A_{11}$ \\
\end{tabular}

We will henceforth make free use of the properties shown in this table. Note also that (12) can be written

$$
\left[A_{10}, A_{10}\right]=\left[A_{01}, A_{01}\right]=0,
$$

and (15) can be written

$$
\left[A_{10}^{2}, A_{11}+A_{01}\right]=\left[A_{01}^{2}, A_{11}+A_{10}\right]=0 .
$$

From (16) we have

$$
\left[A_{01} A_{10}, A_{11}\right]=0 \text {, }
$$

and (19) through (22) specialize to

$$
\begin{aligned}
& a_{11} \cdot x_{10} y_{10}=x_{10} y_{10} \cdot a_{11}=(1 / 2)\left(a_{11} x_{10} \cdot y_{10}+a_{11} y_{10} \cdot x_{10}\right), \\
& a_{11} \cdot x_{01} y_{01}=x_{01} y_{01} \cdot a_{11}=(1 / 2)\left(x_{01} a_{11} \cdot y_{01}+y_{01} a_{11} \cdot x_{01}\right),
\end{aligned}
$$




$$
a_{11}\left(x_{10} y_{01}+y_{01} x_{10}\right)=a_{11} x_{10} \cdot y_{01}+y_{01} a_{11} \cdot x_{10}
$$

and

$$
\left(x_{10} y_{01}+y_{01} x_{10}\right) a_{11}=y_{01} \cdot a_{11} x_{10}+x_{10} \cdot y_{01} a_{11}
$$

respectively.

We assume throughout that $e$ is not a unity element for $A$. We will show

$$
\left(A, A_{11}, A_{11}\right)=\left(A_{11}, A, A_{11}\right)=\left(A_{11}, A_{11}, A\right)=0 .
$$

The submodule $A_{11}$ is a subring of $A$, and for $i \neq j$, two of the associators in $H\left(x_{11}, y_{11}, a_{i j}\right)=\left(x_{11}, y_{11}, a_{i j}\right)+\left(y_{11}, a_{i j}, x_{11}\right)+\left(a_{i j}, x_{11}, y_{11}\right)=0$, are equal to zero, hence all three are equal to zero. Thus it suffices to show that $A_{11}$ is associative.

We assert that the submodule $I=\left(A_{11}, A_{11}, A_{11}\right)+\left(A_{11}, A_{11}, A_{11}\right) A_{11}$ is an ideal of $A$. We will use the fact that the function $T(a, x, y, b)=$ $(a x, y, b)-(a, x y, b)+(a, x, y b)-a(x, y, b)-(a, x, y) b$ is identically zero in any nonassociative ring. Thus $0=T\left(a_{m n}, x_{11}, y_{11}, b_{i j}\right)$, with $m+n=i+j=2$ implies that $A_{11}\left(A_{11}, A_{11}, A_{11}\right) \leqq I$, and with $m+n=2$, $i+j=1$, implies that $\left(A_{11}, A_{11}, A_{11}\right) A_{i j}=0$, using the fact that $\left(A_{11}, A_{11}, A_{i j}\right)=0$. If $m+n=1$ and $i+j=2$ then we get $A_{m n}\left(A_{11}, A_{11}, A_{11}\right)=0$. Thus $\quad\left(A_{11}, A_{11}, A_{11}\right) A+A\left(A_{11}, A_{11}, A_{11}\right)$ is in $I$. Furthermore,

$$
\left(A_{11}, A_{11}, A_{11}\right) A_{11} \cdot A \sqsubseteq\left(\left(A_{11}, A_{11}, A_{11}\right), A_{11}, A\right)+\left(A_{11}, A_{11}, A_{11}\right) A \sqsubseteq I,
$$

so $I A \subseteq I$. Finally,

$$
\begin{aligned}
A \cdot\left(A_{11}, A_{11}, A_{11}\right) A_{11} \subseteq\left(A,\left(A_{11}, A_{11}, A_{11}\right), A_{11}\right) \\
+A\left(A_{11}, A_{11}, A_{11}\right) \cdot A_{11} \subseteq I+I A_{11} \subseteq I,
\end{aligned}
$$

and it follows that $A I \subseteq I$. Hence $I$ is an ideal of $A$. If $A=I$ then $e$ is a unity element for $A$, which contradicts our assumption. Therefore $I=0$ and in particular $\left(A_{11}, A_{11}, A_{11}\right)=0$, which proves (31).

We assert next that $A_{10}^{2}=A_{01}^{2}=0$. First we prove that $J=A_{10}^{2}+$ $A_{10}^{2} A_{10}$ is an ideal of $A$. We have

$$
A_{10} A_{10} \sqsubseteq A_{10} A_{11}=0, \quad A_{01} A_{10}^{2}=A_{10}^{2} A_{01} \subseteq A_{11} A_{01}=0
$$

by using (25), and $A_{11} A_{10}^{2}=A_{10}^{2} A_{11} \subseteq A_{10}^{2}$ by (27). Thus $A_{10}^{2} A+A A_{10}^{2} \sqsubseteq J$. Moreover, $\left(A_{10}^{2} A_{10}\right) A_{11} \subseteq A_{10} A_{11}=0$, and, by using (31), $A_{11}\left(A_{10}^{2} A_{10}\right)=$ $\left(A_{11} A_{10}^{2}\right) A_{10} \subseteq A_{10}^{2} A_{10} \subseteq J$. Letting $a_{11}=u_{10} v_{10}$ in (29) we have

$$
u_{10} v_{10}\left(x_{10} y_{01}+y_{01} x_{10}\right)=\left(u_{10} v_{10} \cdot x_{10}\right) y_{01}+\left(y_{01} \cdot u_{10} v_{10}\right) x_{10}
$$

But $y_{01} \cdot u_{10} v_{10}=u_{10} v_{10} \cdot y_{01}=0$ by using (25), so 


$$
\left(u_{10} v_{10} \cdot x_{10}\right) y_{01}=u_{10} v_{10}\left(x_{10} y_{01}+y_{01} x_{10}\right) \in A_{10}^{2} A_{11} \subseteq J
$$

and therefore $A_{10}^{2} A_{10} \cdot A_{01} \subseteq J$. Finally, $H\left(u_{10} v_{10}, x_{10}, y_{01}\right)=0$ implies

$$
y_{01}\left(u_{10} v_{10} \cdot x_{10}\right)=\left(u_{10} v_{10} \cdot x_{10}\right) y_{01} \in A_{10}^{2} A_{10} \cdot A_{01} \subseteq J .
$$

Thus $J$ is an ideal of $A$.

Since $A$ is simple either $J=0$ or $J=A$. If $J=A$ then $A_{10}^{2}=A_{11}$, $A_{11} A_{10}=A_{10}$ and $A_{01}=0$. Thus we may write

But

$$
e=\sum_{i=1}^{t} x_{10}^{(i)} y_{10}^{(i)}
$$

and

$$
\begin{aligned}
0=H\left(x_{10}, x_{10}, y_{10}\right) & =\left[x_{10}^{2}, y_{10}\right]+\left[x_{10} y_{10}, x_{10}\right]+\left[y_{10} x_{10}, x_{10}\right] \\
& =x_{10}^{2} y_{10}+2 x_{10} y_{10} \cdot x_{10}
\end{aligned}
$$

$$
\begin{aligned}
0= & (1 / 4) P\left(x_{10}, x_{10}, y_{10}, y_{10}\right) \\
= & \left(x_{10}^{2}, y_{10}, y_{10}\right)+2\left(x_{10} y_{10}, x_{10}, y_{10}\right)+2\left(x_{10} y_{10}, y_{10}, x_{10}\right)+\left(y_{10}^{2}, x_{10}, x_{10}\right) \\
= & x_{10}^{2} y_{10} \cdot y_{10}-x_{10}^{2} y_{10}^{2}+2\left(x_{10} y_{10} \cdot x_{10}\right) y_{10}-2 x_{10} y_{10} \cdot x_{10} y_{10}+2\left(x_{10} y_{10} \cdot y_{10}\right) x_{10} \\
& -2 x_{10} y_{10} \cdot y_{10} x_{10}+y_{10}^{2} x_{10} \cdot x_{10}-y_{10}^{2} x_{10}^{2},
\end{aligned}
$$

so using the fact that $A_{10}^{2}$ is in the center of the associative subring $A_{11}$, we obtain $x_{10}^{2} y_{10}^{2}=-2\left(x_{10} y_{10}\right)^{2}$. It follows that

$$
\left(x_{10} y_{10}\right)^{4}=(1 / 4)\left(x_{10}^{2} y_{10}^{2}\right)^{2}=(1 / 4) x_{10}^{4} y_{10}^{4}=0
$$

since $x_{10}^{3}=x_{10} \cdot x_{10}^{2}=0$. But then we have

$$
e=e^{3 t+1}=\left(\sum_{i=1}^{t} x_{10}^{(i)} y_{10}^{(i)}\right)^{3 t+1}=0,
$$

since every term in the multinomial expansion must contain, for some $j$, a factor $\left(x_{10}^{(i)} y_{10}^{(i)}\right)^{4}=0$. From this contradiction we conclude that $J=0$, hence $A_{10}^{2}=0$. Then also $A_{01}^{2}=\left(A_{01}^{\sharp}\right)^{2}=0$, where $A$ is a ring which is anti-isomorphic to $A$.

We may now replace (23) with the table,

\begin{tabular}{c|c|c|c} 
& $A_{11}$ & $A_{10}$ & $A_{01}$ \\
\hline$A_{11}$ & $A_{11}$ & $A_{10}$ & 0 \\
\hline$A_{10}$ & 0 & 0 & $A_{11}$ \\
\hline$A_{01}$ & $A_{01}$ & $A_{11}$ & 0
\end{tabular}.

We will continue to make free use of these multiplicative properties in the sequel. Of special interest are the identities,

$$
y_{01} \cdot z_{01} x_{10}=-z_{01} \cdot x_{10} y_{01}
$$




$$
y_{10} z_{01} \cdot x_{10}=-z_{01} x_{10} \cdot y_{10},
$$

obtained by using the function $H$ and (32).

We show next that the subring $A_{11}$ is itself a simple ring.

Let $B_{11}$ be any nonzero ideal of $A_{11}$ and consider the submodule,

$$
\begin{aligned}
L=B_{11}+ & B_{11} A_{10}+A_{01} B_{11}+A_{01} \cdot B_{11} A_{10} \\
& +A_{01} B_{11} \cdot A_{10}+\left(A_{01} \cdot B_{11} A_{10}\right) A_{11}+A_{11}\left(A_{01} B_{11} \cdot A_{10}\right) .
\end{aligned}
$$

We will show that $L$ is an ideal of $A$.

Evidently, $A B_{11}+B_{11} A \subseteq L$. Also $B_{11} A_{10} \cdot A_{11} \subseteq A_{10} A_{11}=0$; and by (31), $A_{11} \cdot B_{11} A_{10}=A_{11} B_{11} \cdot A_{10} \subseteq B_{11} A_{10} \subseteq L$. By (24),

$$
A_{10} \cdot B_{11} A_{10}=B_{11} A_{10} \cdot A_{10} \subseteq A_{10}^{2}=0 \text {. }
$$

Noting that $B_{11} A_{10} \cdot A_{01} \subseteq A_{01} B_{11} \cdot A_{10}+B_{11} \subseteq L$ by (29), and $A_{01} \cdot B_{11} A_{10} \subseteq L$ by the definition of $L$, we see that $A \cdot B_{11} A_{10}+B_{11} A_{10} \cdot A \subseteq L$. Moreover, $A \cdot A_{01} B_{11}+A_{01} B_{11} \cdot A \subseteq L$ from the left-right symmetry of our identities. Similarly, verification that the fourth and sixth terms in in the definition of $L$ yield elements of $L$ when multiplied on the left or right by an element of $A$ implies the same result for the fifth and seventh terms.

By (26), $\left[A_{01} \cdot B_{11} A_{10}, A_{11}\right] \subseteq\left[A_{01} A_{10}, A_{11}\right]=0$. Since $\left(A_{01} \cdot B_{11} A_{10}\right) A_{11} \subseteq L$ by definition of $L$, it follows that $A_{11}\left(A_{01} \cdot B_{11} A_{10}\right) \subseteq L$ also. Clearly, $A_{10}\left(A_{01} \cdot B_{11} A_{10}\right) \subseteq A_{10} A_{11}=0$, and by (34), $\left(A_{01} \cdot B_{11} A_{10}\right) A_{10} \subseteq A_{10} A_{01} \cdot B_{11} A_{10} \subseteq L$. Also $\left(A_{01} \cdot B_{11} A_{10}\right) A_{01} \subseteq A_{11} A_{01}=0$, and by (30) and (33),

$$
\begin{aligned}
A_{01}\left(A_{01} \cdot B_{11} A_{10}\right) \subseteq & A_{01}\left(B_{11}+A_{10} \cdot A_{01} B_{11}\right) \subseteq L \\
& +A_{01}\left(A_{10} \cdot A_{01} B_{11}\right) \subseteq L+A_{01} B_{11} \cdot A_{01} A_{10} \subseteq L .
\end{aligned}
$$

Thus $A\left(A_{01} \cdot B_{11} A_{10}\right)+\left(A_{01} \cdot B_{11} A_{10}\right) A \subseteq L$.

Since $\left[A_{01} \cdot B_{11} A_{10}, A_{11}\right] \subseteq\left[A_{01} A_{10}, A_{11}\right]=0$ it suffices to show that $\left(A_{01} \cdot B_{11} A_{10}\right) A_{11} \cdot A$ and $A \cdot A_{11}\left(A_{01} \cdot B_{11} A_{10}\right)$ are in $L$. By (31),

$$
\left(A_{01} \cdot B_{11} A_{10}\right) A_{11} \cdot A=\left(A_{01} \cdot B_{11} A_{10}\right) \cdot A_{11} A \subseteq\left(A_{01} \cdot B_{11} A_{10}\right) A \subseteq L
$$

and $A \cdot A_{11}\left(A_{01} \cdot B_{11} A_{10}\right)=A A_{11} \cdot\left(A_{01} \cdot B_{11} A_{10}\right) \subseteq A\left(A_{01} \cdot B_{11} A_{10}\right) \subseteq L$. This completes the verification that $L$ is an ideal of $A$.

Since $A$ is simple and $0 \neq B_{11} \subseteq L$ we must have $L=A$, hence $B_{11} A_{10}=A_{10}$ and $A_{01} B_{11}=A_{01}$.

If $b_{11} \in B_{11}$ then $b_{11}\left(a_{11} x_{10}\right) \cdot y_{01}+y_{01} b_{11} \cdot a_{11} x_{10} \in B_{11}$ and

$$
\left(b_{11} a_{11}\right) x_{10} \cdot y_{01}+y_{01}\left(b_{11} a_{11}\right) \cdot x_{10} \in B_{11}
$$

by (29). Taking the difference of these two elements and using (31) gives $\left(y_{01} b_{11}\right) a_{11} \cdot x_{10}-y_{01} b_{11} \cdot a_{11} x_{10} \in B_{11}$. Since $A_{01} B_{11}=A_{01}$ it follows that $\left(A_{01}, A_{11}, A_{10}\right) \subseteq B_{11}$.

If the intersection of all proper ideals of $A_{11}$ is the zero ideal, 
then $\left(A_{01}, A_{11}, A_{10}\right)=0$. Hence, by (31) and (33),

$$
\begin{aligned}
z_{01}\left(a_{11} \cdot x_{10} y_{01}\right) & =z_{01} a_{11} \cdot x_{10} y_{01}=-y_{01}\left(z_{01} a_{11} \cdot x_{10}\right) \\
& =-y_{01}\left(z_{01} \cdot a_{11} x_{10}\right)=z_{01}\left(a_{11} x_{10} \cdot y_{01}\right) ;
\end{aligned}
$$

i.e., $z_{01}\left(a_{11}, x_{10}, y_{01}\right)=0$.

Since the set $N_{11}$ of elements of $A_{11}$ which annihilate $A_{01}$ is an ideal of $A_{11}$, and since $0=A_{01} N_{11} \neq A_{01}$, it follows that $N_{11}=0$, hence $\left(A_{11}, A_{10}, A_{01}\right)=0$. Thus $A_{10} A_{01}=\left(B_{11} A_{10}\right) A_{01}=B_{11}\left(A_{10} A_{01}\right) \subseteq B_{11}$ and, by using (29), $A_{01} A_{10}=\left(A_{01} B_{11}\right) A_{10} \subseteq B_{11} A_{10} \cdot A_{01}+B_{11} \subseteq B_{11}$. This implies that the ideal $L$ is given by $L=B_{11}+B_{11} A_{10}+A_{01} B_{11}$. Since $A$ is simple, $B_{11}=A_{11}$ and $A_{11}$ is simple.

The other possibility is that $A_{11}$ contains a unique minimal ideal, $M_{11}$. If $\left(A_{01}, A_{11}, A_{10}\right)=0$ we may proceed as above. Thus assume that there exists a nonzero element $b_{11}$ of the form $\left(y_{01}, a_{11}, x_{10}\right)$. Since $\left(A_{01}, A_{11}, A_{10}\right) \subseteq B_{11}$ for every nonzero ideal $B_{11}$ of $A_{11}$, we see that $b_{11} \in M_{11}$. Moreover $b_{11}$ is in the center of $A_{11}$ by (26). Since $M_{11}$ is minimal, $M_{11}=b_{11} A_{11}$. If $b_{11} c_{11}=0$ then, since $A_{01} M_{11}=A_{01}, A_{01} c_{11}=$ $A_{01} A_{11} b_{11} c_{11}=0$. Thus $c_{11} \in N_{11}=0$; i.e., no nonzero element of $A_{11}$ annihilates $b_{11}$. Hence $b_{11}^{2} \neq 0$ and $M_{11}=b_{11}^{2} A_{11}$. Then there exists $b_{11} \in A_{11}$ such that $b_{11}=b_{11}^{2} d_{11}$, or $b_{11}\left(e-b_{11} d_{11}\right)=0$. It follows that $e=b_{11} d_{11} \in M_{11}$ hence $M_{11}=A_{11}$ is simple in this case also.

By (31), (33), and (26), $z_{01}\left(x_{10} y_{01} \cdot a_{11}\right)=\left(z_{01} \cdot x_{10} y_{01}\right) a_{11}=-\left(y_{01} \cdot z_{01} x_{10}\right) a_{11}=$ $-y_{01}\left(z_{01} x_{10} \cdot a_{11}\right)=-y_{01}\left(a_{11} \cdot z_{01} x_{10}\right)=-y_{01} a_{11} \cdot z_{01} x_{10}=z_{01}\left(x_{10} \cdot y_{01} a_{11}\right)$; i.e., $z_{01}\left(x_{10}\right.$, $\left.y_{01}, a_{11}\right)=0$, or $\left(A_{10}, A_{01}, A_{11}\right) \subseteq N_{11}=0$. Then (30) reduces to $y_{01} x_{10} \cdot a_{11}=$ $y_{01} \cdot a_{11} x_{10}$, which, in view of (26), implies that $A_{01} A_{10}$ is an ideal of $A_{11}$. If $A_{01} A_{10}=0$ then (34) implies that $A_{10} A_{01}$ annihilates $A_{10}$, hence $A_{10} A_{01}=$ 0 . But then we easily see from (32) that both $A_{10}$ and $A_{01}$ are ideals of $A$, hence $A_{10}=A_{01}=0$, which implies that $e$ is an unity element for $A=A_{11}$. From this contradiction we conclude that $A_{01} A_{10}=A_{11}$, hence by (26), $A_{11}$ is commutative and therefore a field.

Let $A_{11}=\Phi e$. To prove that $A_{01}$ is one-dimensional over $\Phi$, choose $0 \neq z_{01} \in A_{01}$ such that $z_{01} A_{10}=A_{11}=\Phi e$. Suppose $z_{01} x_{10}=e$. Then for every $y_{01} \in A_{01}$ we have, by (33), $y_{01}=-z_{01} \cdot x_{10} y_{01}=\alpha z_{01}$ for $\alpha \in \Phi$. Also $A_{10}=A_{01}^{\#}$ is one-dimensional over $\Phi$.

We now have $A_{11}=\Phi e, A_{10}=\Phi x$ and $A_{01}=\Phi y$. Since (34) gives $(x y+y x) x=0$ and $x y+y x \in \Phi e$, we must have $x y+y x=0$. Without loss of generality we may take $x y=-y x=e$, which completes the proof of the theorem.

This paper is based on a portion of the authors doctoral dissertation written under the direction of Professor Erwin Kleinfeld, to whom the greatest appreciation is expressed. Thanks are also due the referee who indicated modifications in the original proof allowing for 
an extension to rings of the theorem originally proved only for finitedimensional algebras.

\section{BIBLIOGRAPHY}

1. A. A. Albert, Power-associative rings, Trans. Amer. Math. Soc. 64 (1948), 552-593.

2. Almost alternative algebras, Portugaliae Math. 8 (1949), 23-36.

3. E. Kleinfeld, Rings of $(\gamma, \delta)$ type, Portugal Math. 18 (1959), 107-110.

4. Simple algebras of type $(1,1)$ are associative, Canad. J. Math. 13 (1961), 129-148.

5. L. A. Kokoris, A class of almost alternative algebras, Canad. J. Math. 8 (1956), 250-255.

6. - On rings of $(\gamma, \delta)$ type, Proc. Amer. Math. Soc. 9 (1958), 897-904.

7. F. Kosier, On a class of non-flexible algebras, Trans. Amer. Math. Soc. 102 (1962) 299-318.

8. C. Maneri, Simple $(-1,1)$ rings with an idempotent, Proc. Amer. Math. Soc. 14 (1963), 110-117.

9. L. M. Weiner, Lie admissible algebras, Univ. Nac. Tucuman Rev. Ser. A. 11 (1957), 10-24.

Received March 21, 1968, and in revised form September 17, 1968.

Wright State University

DAYTON, OHIO 


\section{PACIFIC JOURNAL OF MATHEMATICS}

\section{EDITORS}

H. ROYDEN
Stanford University
Stanford, California

\section{R. R. PHELPS}

University of Washington

Seattle, Washington 98105
J. DUGUNDJI

Department of Mathematics

University of Southern California

Los Angeles, California 90007

\section{RICHARD ARENS}

University of California

Los Angeles, California 90024

\section{ASSOCIATE EDITORS}

E. F. BeCKenbaCh
B. H. NEUMANN

F. WOLF
K. YoshidA

\section{SUPPORTING INSTITUTIONS}

\author{
UNIVERSITY OF BRITISH COLUMBIA \\ CALIFORNIA INSTITUTE OF TECHNOLOGY \\ UNIVERSITY OF CALIFORNIA \\ MONTANA STATE UNIVERSITY \\ UNIVERSITY OF NEVADA \\ NEW MEXICO STATE UNIVERSITY \\ OREGON STATE UNIVERSITY \\ UNIVERSITY OF OREGON \\ OSAKA UNIVERSITY \\ UNIVERSITY OF SOUTHERN CALIFORNIA
}

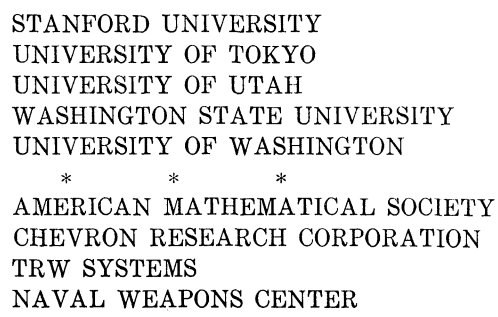

The Supporting Institutions listed above contribute to the cost of publication of this Journal, but they are not owners or publishers and have no responsibility for its content or policies.

Mathematical papers intended for publication in the Pacific Journal of Mathematics should be in typed form or offset-reproduced, double spaced with large margins. Underline Greek letters in red, German in green, and script in blue. The first paragraph or two must be capable of being used separately as a synopsis of the entire paper. It should not contain references to the bibliography. Manuscripts, in duplicate if possible, may be sent to any one of the four editors. Please classify according to the scheme of Math. Rev. 36, 1539-1546. All other communications to the editors should be addressed to the managing editor, Richard Arens, University of California, Los Angeles, California, 90024.

50 reprints are provided free for each article; additional copies may be obtained at cost in multiples of 50 .

The Pacific Journal of Mathematics is published monthly. Effective with Volume 16 the price per volume (3 numbers) is $\$ 8.00$; single issues, $\$ 3.00$. Special price for current issues to individual faculty members of supporting institutions and to individual members of the American Mathematical Society: $\$ 4.00$ per volume; single issues $\$ 1.50$. Back numbers are available.

Subscriptions, orders for back numbers, and changes of address should be sent to Pacific Journal of Mathematics, 103 Highland Boulevard, Berkeley, California, 94708.

PUBLISHED BY PACIFIC JOURNAL OF MATHEMATICS, A NON-PROFIT CORPORATION

Printed at Kokusai Bunken Insatsusha (International Academic Printing Co., Ltd.), 7-17, Fujimi 2-chome, Chiyoda-ku, Tokyo, Japan. 


\section{Pacific Journal of Mathematics \\ Vol. 29, No. $2 \quad$ June, 1969}

Bruce Langworthy Chalmers, On boundary behavior of the Bergman kernel function and related domain functionals ................... 243

William Eugene Coppage, Peirce decomposition in simple Lie-admissible power-associative rings .............................. 251

Edwin Duda, Compactness of mappings...................... 259

Earl F. Ecklund Jr., On prime divisors of the binomial coefficient......... 267

Don E. Edmondson, A modular topological lattice ............... 271

Phillip Alan Griffith, A note on a theorem of Hill ................... 279

Marcel Herzog, On finite groups with independent cyclic Sylow

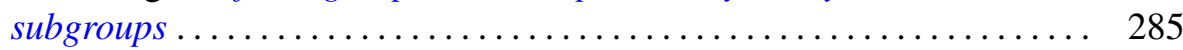

James A. Huckaba, Extensions of pseudo-valuations................. 295

S. A. Huq, Semivarieties and subfunctors of the identity functor ........ 303

I. Martin (Irving) Isaacs and Donald Steven Passman, Finite groups with small character degrees and large prime divisors. II ............ 311

Carl Kallina, A Green's function approach to perturbations of periodic

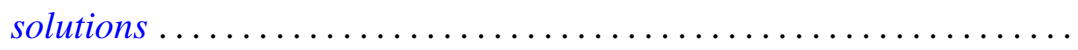

$\mathrm{Al}$ (Allen Frederick) Kelley, Jr., Analytic two-dimensional subcenter manifolds for systems with an integral ....................

Alistair H. Lachlan, Initial segments of one-one degrees ............ 351

Marion-Josephine Lim, Rank k Grassmann products ............. 367

Raymond J. McGivney and William Henry Ruckle, Multiplier algebras of

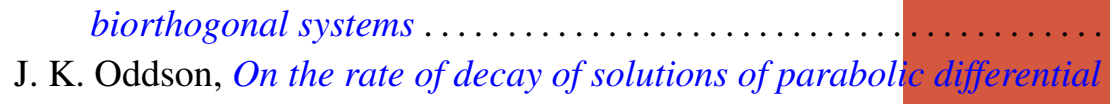

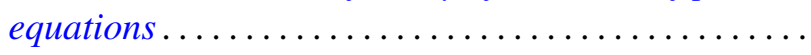

Helmut R. Salzmann, Geometries on surfaces ........... .

Annemarie Schlette, Artinian, almost abelian groups and their groups of automorphisms ............................

Edgar Lee Stout, Additional results on modules over polydisc algebras ...

Lajos Tamássy, A characteristic property of the sphere . .

Mark Lawrence Teply, Some aspects of Goldie's torsion theory. ...

Freddie Eugene Tidmore, Extremal structure of star-shaped sets ...

461

Leon Jarome Weill, Unconditional and shrinking bases in locally convex spaces... 